\title{
Diagnostic sensitivity of impulse oscillometry in early detection of patients exposed to risk factors chronic obstructive pulmonary diseases
}

\author{
Seyed Ali Javad Mousavi ${ }^{1}$, Jafar Aslani ${ }^{2}$, Zahra Aslani ${ }^{3}$, Hanieh Raji ${ }^{4 *}$ \\ Received: 21 Aug 2019 \\ Published: 12 Jul 2021
}

\section{Abstract}

Background: Impulse oscillometry (IOS) is a method that does not depend on the cooperation of the patient and can detect small airway diseases with higher sensitivity than spirometry. However, the clinical application value of IOS in the screening of patients exposed to risk factors COPD and early diagnosis remains unclear. The aim of this study is to evaluate diagnostic sensitivity of IOS in the early detection of patients exposed to risk factors COPD.

Methods: A prospective cross-sectional study was conducted in Rasoul Akram Hospital, Tehran, Iran, from 2013 to 2015.28 patients with COPD risk factors and normal spirometry participated in the study. The IOS was performed. We obtained the respiratory resistance and impedance of $5 \mathrm{~Hz}$ (R5) and $20 \mathrm{~Hz}$ (R20) and $5 \mathrm{~Hz}$ (Z5), respectively. The data were analyzed using SPSS version 17 using Chi-square and two independent sample t-test. Spearman correlation test was used to measure the correlation of oscillometry parameters in the diagnosis of COPD. P-value $<0.05$ was considered significantfor all statistical analyses.

Results: The mean patient age was $55.50 \pm 11.27$ years. In this study, the sensitivity of Z5, R5, and R20 was respectively $28.5 \%$, $25 \%$, and $31.5 \%$. All oscillometry parameters were significantly correlated with each other but none of the oscillometry parameters showed significant correlations with $\mathrm{FEV}_{1} / \mathrm{FVC}(\mathrm{rZ5}=0.018, \mathrm{rR} 5=0.082, \mathrm{rR} 20=0.041$ and $\mathrm{PZ5}=0.932, \mathrm{PR} 5=0.711, \mathrm{P} \mathrm{R} 20=0.850)$. According to the results, only 9 patients (32.5\%) with normal values of $\mathrm{FEV}_{1} / \mathrm{FVC}$ had abnormal values of oscillometry.

Conclusion: IOS has a low sensitivity and cannot be used in the screening of early-stage chronic obstructive pulmonary disease.

Keywords: COPD, Impulse oscillometry, Spirometry, Airway obstruction

Conflicts of Interest: None declared

Funding: None

\section{*This work has been published under CC BY-NC-SA 1.0 license.}

Copyright $\odot$ Iran University of Medical Sciences

Cite this article as: Mousavi SAJ, Aslani J, Aslani Z, Raji H. Diagnostic sensitivity of impulse oscillometry in early detection of patients exposed to risk factors chronic obstructive pulmonary diseases. Med J Islam Repub Iran. 2021 (12 Jul);35:89. https://doi.org/10.47176/mjiri.35.89

\section{Introduction}

An enormous public health problem, chronic obstructive pulmonary disease (COPD) is marked by airflow limitation. COPD is reported as the fourth cause of death around the world and was expected to rank as the third cause of mortality by $2020(1,2)$.

The main objective of any COPD control program is early diagnosis. This can allow more time for patients to react to disease progression. COPD patients predominantly suffer respiratory sy mptoms like sputum production and cough, and dyspnea. However, it is not clear how respiratory symptoms play a role in the early diagnosis of

Corresponding author: Dr Hanieh Raji, raji-h@ajums.ac.ir

1. Air Pollution Research Center, Iran University of Medical Sciences, Tehran, Iran

2. Research Center for Chemical Injuries, Baqiyatallah Medical Sciences University, Tehran, Iran

3. Faculty of Medicine, Iran University of Medical Sciences, Tehran, Iran

4. Air Pollution and Respiratory Diseases Research Center, Ahvaz Jundishapur University of Medical Sciences, Ahvaz, Iran
COPD. Patients may not adequately report respiratory symptoms. Moreover, the relationship between respiratory symptoms and spirometric parameters is not strong $(3,4)$. Spirometry is the most common test for measuring pulmonary function, diagnosing and following COPD. However, the test is not the most sensitive one. It perhaps has shortcomings in identifying COPD at its early stages (5).

Impedance oscillometry (IOS) is a method for the diagnosis of pulmonary diseases that measures the resistance of airways using sound waves and detects airway diseases through the diagnosis of changes in the resistance of air-

$\uparrow$ What is "already known" in this topic:

Impulse oscillometry (IOS) is a method that can detect small airway diseases with higher sensitivity than spirometry. However, the clinical application value of IOS in the early detection of suspected COPD patients remains unclear.

$\rightarrow$ What this article adds:

IOS has a low sensitivity for early detection of COPD patients. 
ways. This method is not dependent on patient's cooperation and is more sensitive in the diagnosis of obstructive lesions to the small airways compared to previous methods. Therefore, it can be helpful for elderly patients and those with physical or mental limitations (6). Impedance oscillometry has been reported to be abnormal in patients with pulmonary symptoms and normal $\mathrm{FEV}_{1}$ in spirometry (4). Borrill et al. have studied IOS and specific airway conductance comparing $\mathrm{FEV}_{1}$ in patients with COPD. Their sample included patients who experienced subtherapeutic doses of bronchodilators (short- and long-acting). The results showed that the IOS method performed better in identifying more delicate changes in pulmonary function compared to the conventional spirometry $(7,8)$. In the same vein, numerous researches have done on impulse oscillometry and its role in detecting abnormalities in patients with COPD symptoms who had not yet shown abnormal spirometry $(4,9)$. Therefore, these complementary diagnostic techniques are unlikely to replace spirometry but have the potential to help physicians to diagnose COPD at the early stages. This is particularly true about patients with symptoms that are disproportionate to spirometry (6). In this study, it was assumed that using the IOS method, the individuals at high risk for COPD that are prone to COPD and its complications could be identified which is a crucial step for early diagnosis of COPD patients.

\section{Methods}

This cross-sectional study was conducted on 28 patients who were admitted to the pulmonary clinic in Rasoul Akram Hospital, Tehran, Iran. This research was approved by the Institutional Ethical Review Board at Iran University of Medical Sciences (IUMS). The patients were selected by a non-probability and availability sampling method. All participants provided written informed consent. The subjects who had chronic respiratory symptoms, including cough, sputum and dyspnea, and exposure to the risk factor of COPD such as smoking or occupational exposure to pollutants and their spirometry results were normal $\left(\mathrm{FEV}_{1}>80\right.$ and $\left.\mathrm{FEV}_{1} / \mathrm{FVC}>75 \%\right)$ were included. Patients with other respiratory diseases such as bronchiectasis, chronic allergic sinusitis and interstitial lung diseases were excluded. The research recorded demographic data con- sisting of: gender, age, height, weight, smoking, body mass index (BMI), work history, and respiratory symptoms. The IOS was measured in the patients using a device in a private clinic (Master lab-IOS, Erich Jaeger, Germany). The IOS parameters were the respiratory resistance and respiratory impedance at $5 \mathrm{~Hz}$ (R5) and 20 $\mathrm{Hz}(\mathrm{R} 20)$ and $5 \mathrm{~Hz}(\mathrm{Z} 5)$, respectively. In addition, the spirometry assessment was performed using the Masterlab Pro unit (1051108-175101, Jaeger, Germany) which was calibrated at the start of each diay. The spirometry parameters of $\mathrm{FEV}_{1}, \mathrm{FVC}$, ERV, PEF and MMEF75-25 were measured and recorded in all patients. Parameters were measured through oral in a sitting position within normal breathing that given the normal range, the resistance percentage of airways was interpreted into two categories of abnormal and normal by a pulmonologist. The raw results of spirometry were normalized based on age and gender with Predicted amounts of healthy subjects. If we consider the cut-off point of $100 \%$ for all these parameters, since all patients entering the study had the FEV1/FVC values of $75 \%$ that was the normal value, the accuracy of detection for each of the existing guideline equivalent impedance of $150 \%$ was normal. The diagnostic sensitivity for all three frequencies in this study was calculated according to this approach.

The data were analyzed with SPSS version 17 using Chi-square and two independent sample t-test. The Spearman correlation test was used to investigate the correlation of oscillometry prameters in diagnosis of COPD.The p-value of less than 0.05 was considered statistically significant for statistical analyses in the study.

\section{Results}

Twenty-eight patients (21 males and 7 females) were enrolled in this study. The mean age of patients was $55.50 \pm 11.72$ years. 19 patients $(67.8 \%)$ were smoker and $9(32.2 \%)$ were non-smoker. The mean height and weight of patients were $170 \pm 10.0 \mathrm{~cm}$ and $77.77 \pm 14.05 \mathrm{~kg}$, respectively.

Results of oscillometry and spirometry analysis according to gender and smoking status are shown in Tables 1 and 2. Only the Height was significantly different between the two gender groups $(p=0.001)$ (Table 1). The Height variable was significantly different

Table 1. Demographic characteristics, spirometry, and oscillometry in all patients according to gender

\begin{tabular}{lcccc}
\hline Variable & Total & Male & Female & $\mathrm{p}$ \\
\hline Age & $55.50 \pm 11.27$ & $55.33 \pm 12.51$ & $56.00 \pm 9.83$ & 0.85 .4 \\
Weight (Kg) & $77.77 \pm 14.05$ & $78.40 \pm 15.14$ & $76.00 \pm 11.12$ & 0.676 \\
Height (m) & $1.70 \pm 0.10$ & $1.72 \pm 0.10$ & $1.61 \pm 0.06$ & $0.001^{*}$ \\
FVC (\%) & $95.69 \pm 23.03$ & $93.72 \pm 24.37$ & $101.04 \pm 19.57$ & 0.3915 \\
FEV (\%) & $95.72 \pm 15.36$ & $93.31 \pm 13.57$ & $102.40 \pm 18.72$ & 0.22 .0 \\
PEFV(\%) & $87.28 \pm 30.08$ & $78.31 \pm 23.99$ & $110.32 \pm 33.58$ & 0.055 \\
MMEF75-25 (\%) & $88.36 \pm 26.44$ & $88.85 \pm 28.94$ & $86.98 \pm 19.83$ & 0.812 \\
ERV (\%) & $122.76 \pm 88.73$ & $102.44 \pm 42.44$ & $153.23 \pm 131.55$ & 0.6017 \\
Z5 (\%) & $120.92 \pm 49.80$ & $114.01 \pm 36.73$ & $140.65 \pm 76.57$ & 0.341 \\
R5 (\%) & $116.14 \pm 50.32$ & $110.16 \pm 40.55$ & $132.37 \pm 72.08$ & 0.4917 \\
R20 (\%) & $106.83 \pm 38.98$ & $104.88 \pm 31.80$ & $112.40 \pm 57.73$ & 0.6815 \\
FEV $/$ FVC & $78.34 \pm 9.6$ & $79.52 \pm 5.7$ & $77.6 \pm 6.4$ & 0.615 \\
\hline *Normal distributed & & & &
\end{tabular}

*Normal distributed variable

Data are expressed as Mean \pm SD unless otherwise stated.

Two independent sample t-test 
Table 2. Comparison of demographic characteristics, spirometry, and oscillometry in patients, according to smoking

\begin{tabular}{lcccc}
\hline Variable & Total & Non-Smokers & Smokers & $\mathrm{p}$ \\
\hline Age & $55.50 \pm 11.27$ & $58.58 \pm 10.54$ & $53.19 \pm 12.35$ & 0.111 \\
Weight & $77.77 \pm 14.05$ & $77.09 \pm 11.57$ & $78.25 \pm 15.88$ & 0.946 \\
Height & $1.70 \pm 0.10$ & $1.65 \pm 0.06$ & $1.73 \pm 0.11$ & $0.001 *$ \\
FVC (\%) & $95.69 \pm 23.03$ & $90.45 \pm 19.95$ & $98.97 \pm 25.72$ & 0.395 \\
FEV (\%) & $95.72 \pm 15.36$ & $95.35 \pm 19.95$ & $95.98 \pm 12.18$ & 0.220 \\
PEF (\%) & $87.28 \pm 30.08$ & $82.48 \pm 33.71$ & $90.48 \pm 18.16$ & 0.055 \\
MMEF75-25 (\%) & $88.36 \pm 26.44$ & $88.75 \pm 34.38$ & $88.16 \pm 22.53$ & 0.812 \\
ERV (\%) & $122.76 \pm 88.73$ & $104.00 \pm 21.70$ & $127.45 \pm 99.07$ & 0.607 \\
Z5 (\%) & $120.92 \pm 49.80$ & $130.80 \pm 35.83$ & $114.13 \pm 57.64$ & 0.341 \\
R5 (\%) & $116.14 \pm 50.32$ & $131.64 \pm 39.68$ & $106.45 \pm 54.91$ & 0.497 \\
R20 (\%) & $106.83 \pm 38.98$ & $122.12 \pm 32.79$ & $96.31 \pm 40.33$ & 0.685 \\
FEV $/$ FVC & $78.34 \pm 9.6$ & $80.25 \pm 5.3$ & $77.71 \pm 6.8$ & 0.615 \\
\hline *Normal distributed variable & & & &
\end{tabular}

*Normal distributed variable

Data are expressed as Mean \pm SD unless otherwise stated.

Two independent sample t-test

between the smoker and non-smoker groups $(\mathrm{p}=0.001)$ (Table 2).

The correlations between the parameters of oscillometry and also between these parameters and FEV1/FVC were assessed (Tables 3 and 4). The results showed that R5 was significantly correlated with Z5 $(\mathrm{r}=0.926, \mathrm{p}<0.001)$ and R20 was significantly correlated with Z5 ( $\mathrm{r}=0.882$, $\mathrm{p}<0.001)$. The strength of the correlations between R20 and R5 showed a significant association between their measurements $(\mathrm{r}=0.949, \mathrm{p}<0.001)$.

In conclusion, the results showed that all oscillometry parameters were significantly correlated with each other. The correlation between oscillometry parameters (Z5, R5, R20) and $\mathrm{FEV}_{1} / \mathrm{FVC}$ was also calculated. None of the oscillometry parameters showed significant correlations with FEV1/FVC $\left(\mathrm{r}_{\mathrm{Z} 5}=0.018, \mathrm{r}_{\mathrm{R} 5}=0.082, \mathrm{r}_{\mathrm{R} 20}=0.041\right.$ and $\left.\mathrm{P}_{\mathrm{Z} 5}=0.932, \mathrm{P}_{\mathrm{R} 5}=0.711, \mathrm{P}_{\mathrm{R} 20}=0.850\right)$.

According to the results, only 9 patients (32.5\%) with normal values of FEV1/FVC had abnormal values of oscillometry (Table 5).

\section{Discussion}

In this study, the diagnostic sensitivity rate of IOS parameters was evaluated in patients with chronic respiratory symptoms, including cough, sputum and dyspnea, and exposure to the risk factor of COPD. The IOS parameters were total resistance of respiratory system $(Z)$ and resistance airway and lung tissue (R). The results showed that the sensitivity of Z5, R5, and R20 was $28.5 \%$, $25 \%$ and $31.5 \%$, respectively. In the current study, R20 and R5 had the highest and least sensitivities in the early diagnosis of COPD, respectively.

Several studies have shown that IOS is a valuable technique for the diagnosis of early stages of COPD. In a study by Franz et al. in Sweden conducted on 450 cases, IOS had a high value in the earlier diagnosis of COPD. In the present study, chronic bronchitis, asthma or COPD were related to lower pulmonary reactance and higher pulmonary resistance (4).

Winkler et al. conducted a study on 244 patients with asthma and chronic bronchitis and reported that all parameters of IOS except for R20 are effective in the diagnosis of moderate and mill obstruction, while severe obstructions are recognizable by frequency dependence of resistance (FDR) and reactance in $5 \mathrm{~Hz}$ and $\mathrm{X} 5$. Therefore, IOS is a suitable method for the evaluation of airway obstruction in patients with low cooperation; in addition, it is helpful as a sensitive screening tool for the early diagnosis of bronchial obstruction (10). The lower sensitivity of IOS in our study can be related to fewer patients and type of patients (at-risk patients). AL-Mutairi et al. conducted a study on COPD patients and healthy subjects and reported that the sensitivity for patients and healthy subjects was $38.95 \%$ and $45.8 \%$, respectively. The results of this study were almost the same as those of ours

Table 3. The correlation of important oscillometry parameters in early diagnosis of COPD

\begin{tabular}{lcc}
\hline Parameters & Pearson Correlation & $\mathrm{p}$ \\
\hline Z5 \& R5 & 0.926 & $<0.001$ \\
Z5 \& R20 & 0.882 & $<0.001$ \\
R5 \& R20 & 0.949 & $<0.001$ \\
\hline
\end{tabular}

Table 4. The correlation of oscillometry parameters with FEV1/FVC

\begin{tabular}{lcc}
\hline Parameters & Pearson Correlation & $\mathrm{p}$ \\
\hline $\mathrm{FEV}_{1} / \mathrm{FVC} \&$ Z5 & 0.018 & 0.932 \\
$\mathrm{FEV}_{1} / \mathrm{FVC} \&$ R20 & 0.041 & 0.850 \\
$\mathrm{FEV}_{1} / \mathrm{FVC}$ \& R5 & 0.82 & 0.711 \\
\hline
\end{tabular}

Table 5. The diagnostic sensitivity rate of IOS parameters in early determination COPD patients

\begin{tabular}{lcc}
\hline Parameters & Number $(\mathrm{n})$ & Sensitivity $(\%)$ \\
\hline Z5 & 8 & 28.5 \\
R5 & 7 & 25 \\
R20 & 5 & 31.5 \\
AAt least one $>150 \%$ & 9 & $32 \%$ \\
All $>105 \%$ & 4 & 14.5 \\
\hline
\end{tabular}


(11).

Nikkhah et al. examined IOS parameters on COPD patients. These results reported that the minimum and maximum sensitivity belonged to R20 and X5, which is not consistent with the results of our study (12).

Kolsum et al. evaluated the results of IOS and spirometry performed on 58 patients with COPD and followed them for a year. They reported that IOS is not more sensitive than $\mathrm{FEV}_{1}$ and cannot be used as an alternative for $\mathrm{FEV}_{1}$ (13); however, other researchers reported that IOS is more sensitive than $\mathrm{FEV}_{1}(14,15)$.

In COPD patients, there has been a significant lag between the development of small airway diseases to the onset of $\mathrm{FEV}_{1}$ decline. Some at-risk patients such as heavy smokers, may have developed small airway diseases; this can be a clue for proper detection of patients with early-stage COPD may have clinical importance (16). Some studies have documented that IOS parameters showed more sensitivity in identifying SADs among people exposed to environmental hazards and asymptomatic heavy-smokers. Therefore, IOS can be more sensitive in identifying early-stage COPD $(17,18)$.

In our study and some other studies, the R20 parameter had the most sensitivity among other parameters of IOS. Studies have shown that compared to the resistance, reactance is more appropriate to investigate the intensity and degree of airway obstruction and peripheral airway obstruction in COPD patients but it is an inadequate parameter to assess the mechanical changes in airways . Qi et al. demonstrated that IOS parameters (R5, R10, R5-20, Z5 and Fres) and area of reactance had an inverse correlation with FEV1 and PEF. Meanwhile, reactance at $5 \mathrm{~Hz}$ had a positive correlation with FEV1 and PEF. Increased levels of R5, R10, R20, Z5, Fres and reactance was related to the increase in the stage of obstruction (19), whereas the study by Kolsum et al. reported that IOS parameters R5, X5 and resonant frequency (Fres) were significantly correlated with $\mathrm{FEV}_{1}, \mathrm{TLC}$ and RV while R20 was significantly correlated with RV. Also resistance (R) was more appropriate for airway obstruction, especially for peripheral airways (13).

Therefore, by examining and considering the correlation between parameters of oscillometry and spirometry and the sensitivity rate of oscillometry parameters, it can be concluded that the IOS test cannot be considered as a good alternative for spirometry, and it cannot be used in screening for COPD patients. Another result of our study was that the resistance parameters (R5, R20 and Z5) in females were more than those in males; however, there was no significant difference in this regard which was consistent with the results obtained by Schulz et al. (20). In the current study, airway resistance (R5, R20 and Z5) and spirometry volumes $\left(\mathrm{FEV}_{1}, \mathrm{FVC}, \mathrm{VT}, \mathrm{FEV}_{1} / \mathrm{FVC}, \mathrm{ERV}, \mathrm{MMEF} 25-75\right.$ and PEF) reflected no statistically significant differences between smokers and non-smokers. In other words, in those who have chronic respiratory symptoms and FEV1 has not declined yet, smoking does not make any difference in lung volumes and airway resistance.

Our study Limitation is the low sample size, and there is no control group. It is better to measure air trapping in chest CT scan and investigate the relationship with IOS data. Also, the diversity of the age of the patients is the limitation of the study that perhaps are the reason for the low sensitivity of IOS and weak correlation of parameters of IOS and spirometry in these patients. Therefore, doing further studies with a greater sample size can bring about more accurate and acceptable results.

Conclusion

Although in other studies, IOS has been discussed as an appropriate method for the evaluation of airway obstruction disease with the minimal dependency on patient cooperation, the findings of the present study showed that this method has a low sensitivity in the early diagnosis of COPD and cannot be used in screening for chronic obstructive pulmonary disease.

Conflict of Interests
The authors declare that they have no competing interests.

References

1. Brij SO, Chatterji S, Marquette M. Chronic Obstructive Pulmonary Disease (COPD). Clinical Pathways in Emergency Medicine: Springer; 2016. p. 245-57.

2. Mannino DM, Buist AS. Global burden of COPD: risk factors, prevalence, and future trends. Lancet. 2007;370(9589):765-73.

3. de Marco R. Accordini S. Cerveri I. Corsico A. Anto J.M. Kunzli N. et al.Incidence of chronic obstructive pulmonary disease in a cohort of young adults according to the presence of chronic cough and phlegm. Am J Respir Crit Care Med. 2007;175:32-39.

4. Frantz S, Nihlén U, Dencker M, Engström G, Löfdahl CG, Wollmer P. Impulse oscillometry may be of value in detecting early manifestations of COPD. Respir Med. 2012;106(8):1116-23. 5. Csikesz NG, Gartman EJ. New developments in the assessment of COPD: early diagnosis is key. Int $\mathrm{J}$ Chronic Obstruct Pulm Dis. 2014;9:277-286.

6. Oostveen E, MacLeod D, Lorino HI, Farre R, Hantos Z, Desager K, et al. The forced oscillation technique in clinical practice: methodology, recommendations and future developments. Eur Respir J. 2003;22(6):1026-41.

7. Borrill ZL, Houghton CM, Tal-Singer R, Vessey SR, Faiferman I, Langley SJ, et al. The use of plethysmography and oscillometry to compare long-acting bronchodilators in patients with COPD. Br J Clin Pharmacol. 2008;65(2):244-252.

8. Borrill Z, Houghton C, Woodcock A, Vestbo J, Singh D. Measuring Bronchodilation In COPD Clinical Trials. Br J Clin pharmacol. 2005;59(4):379-84.

9. Oppenheimer BW, Goldring RM, Berger KI. Distal airway function assessed by oscillometry at varying, respiratory rate: comparison with dynamic compliance. COPD. 2009;6(3):162-170.

10. Winkler J, Hagert-Winkler A, Wirtz H, Schauer J, Kahn T, Hoheisel G. [Impulse oscillometry in the diagnosis of the severity of obstructive pulmonary disease]. Pneumologie (Stuttgart, Germany). 2009;63(5):266-75.

11. Al-Mutairi S, Sharma $\mathrm{P}$, Al-Alawi A, Al-Deen J. Impulse oscillometry: an alternative modality to the conventional pulmonary function test to categorise obstructive pulmonary disorders. Clin Exp Med. 2007;7(2):56-64.

12. Nikkhah M, Amra B, Eshaghian A, Fardad S, Asadian A, Roshanzamir T, et al. Comparison of impulse osillometry system and spirometry for diagnosis of obstructive lung disorders. Tanaffos. 2011;10(1):19.

13. Kolsum U, Borrill Z, Roy K, Starkey C, Vestbo J, Houghton C, et al. Impulse oscillometry in COPD: identification of measurements related to airway obstruction, airway conductance and lung volumes. Respir Med. 2009;103(1):136-43.

14. Houghton C, Woodcock A, Singh D. A comparison of plethysmography, spirometry and oscillometry for assessing the pulmonary effects of inhaled ipratropium bromide in healthy subjects and patients with asthma. Br J Clin Pharmacol. 2005;59(2):152-9.

15. Houghton CM, Woodcock AA, Singh D. A comparison of lung function methods for assessing dose-response effects of salbutamol. Br J Clin Pharmacol. 2004;58(2):134-41.

16. Su ZQ, Guan WJ, Li SY, Ding M, Chen Y, Jiang M, et al. Significances of spirometry and impulse oscillometry for detecting small airway disorders assessed with endobronchial optical coherence tomography in COPD. Int J Chron Obstruct Pulmon Dis. 2018 Oct:13:3031-44.

17. Skloot G, Goldman M, Fischler D, Goldman C, Schechter C, Levin $\mathrm{S}$, et al. Respiratory symptoms and physiologic assessment of ironworkers at the World Tradle Center disaster site. Chest. 2004;125(4):1248-1255.

18. Oppenheimer BW, Goldring RM, Herberg ME, Hofer IS, Reyfman PA, Liautaud S, et al. Distal airway/ function in symptomatic subjects with normal spirometry following World Trade Center dust exposure. Chest. 2007;132(4):1275-1282.

19. Qi GS, Zhou ZC, Gu WC, Xi F, Wu H, Yang WL, et al. Detection of the airway obstruction stage in asthma using impulse oscillometry system. J Asthma. 2013;50(1):45-51.

20. Schulz H, Flexeder C, Behr J, Heier M, Holle R, Huber RM, et al. Reference values of impulse oscillometric lung function indices in adults of advanced age. PloS One. 2013;8(5):e63366. 